\title{
ELECTRON TRANSPORT IN SUBMICRON WIRES OF SEMICONDUCTORS
}

\author{
J. WRÓBEL \\ Institute of Physics, Polish Academy of Sciences \\ Al. Lotników 32/46, 02-668 Warszawa, Poland
}

\begin{abstract}
We review the methods of fabrication and transport properties of submicron II-VI, IV-VI and III-V semiconductor wires. Devices were prepared by electron-beam lithography and used for detailed magnetotransport studies, carried out at low (down to $30 \mathrm{mK}$ ) temperatures. We discuss a number of novel features obtained in ballistic, diffusive and localized transport regimes. In particular, we describe the universal conductance fluctuations for semimagnetic materials (CdMnTe) and discuss the edge cliannel transport for $\mathrm{PbTe}, \mathrm{PbSe}$ and $\mathrm{GaAs} / \mathrm{GaAlAs}$ systems.
\end{abstract}

PACS numbers: 72.15.Rn, 73.20.Fz, 73.61.Ga, 75.50.Pp

\section{Introduction}

Electron-beam (e-beam) lithography offers flexibility and resolution necessary to make a submicron devices from virtually any material [1]. This gives the opportunity to study the quantum nature of dimensionality-dependent electron diffusion in solids. The most spectacular examples of such effects are unexpectedly large conductance fluctuations in small samples [2] and conductance quantization observed in point contacts at low temperatures $[3,4]$. In this article we review the transport studies of submicron semiconductor wires fabricated in the Nanostructure Laboratory, operated jointly by the Institute of Physics, Polish Academy of Sciences, and the Institute of Electron Technology in Warsaw. For semimagnetic wires we demonstrated the existence of a new driving mechanism of conductance fluctuations induced by the giant $s-d$ exchange spin splitting [5]. We observed an enhanced conductivity of the sample edges in the IIall bridges patterned from $\mathrm{PbSe}$ and $\mathrm{PbTe}$ epilayers - an effect caused by the strain-induced carrier redistribution between the energy valleys [6]. We studied also the two-terminal conductance quantization in GaAs/GaAlAs heterostructure wires. We confirmed experimentally the role of a total electrostatic energy [7] for the edge currents formation in high magnetic fields. 


\section{Fabrication methods and materials}

We used Jeol 6400 scanning electron microscope (with maximum accelerating voltage $40 \mathrm{keV}$ and low current beam diameter $4 \mathrm{~nm}$ ) as an e-beam writing machine. Scanning coils of the microscope were controlled by the Raith Elphy Plus system with vectorscan writing, videobackground capturing and alignment mark recognition abilities. We used also the laser interferometer controlled stage with positioning resolution $5 \mathrm{~nm}$ and multilevel overlay accuracy $<100 \mathrm{~nm}$.

The six-terminal Hall bars and two-terminal (single and multiple) quantum wires were formed with $30 \mathrm{keV}$, proximity corrected exposure. Devices were defined by wet etching or dry etching (Ar ion milling) with e-beam resist used as the etching mask. The geometrical widths of patterned wires $W_{\text {geo }}$ ranged from 0.3 to $1 \mu \mathrm{m}$. Figure 1 shows few examples of the fabricated structures.

a)

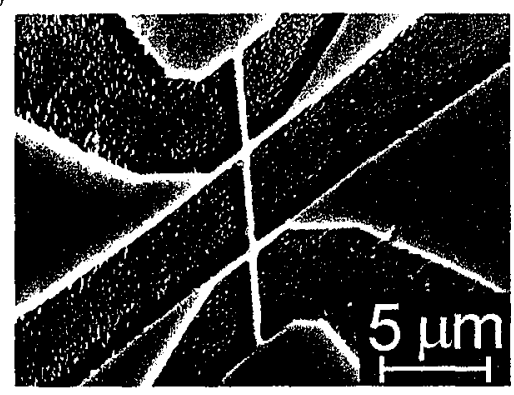

(i)

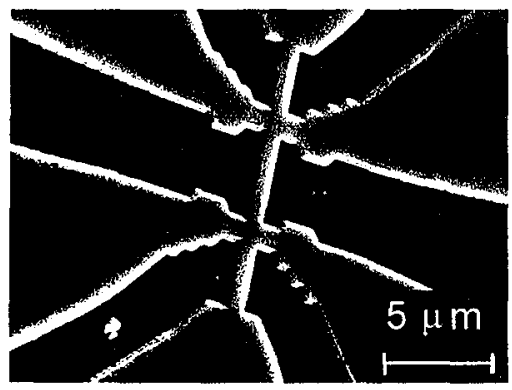

b)

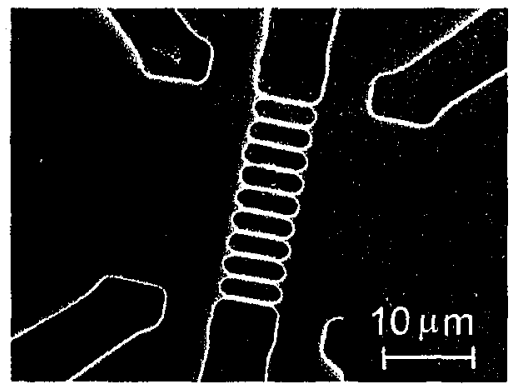

d)

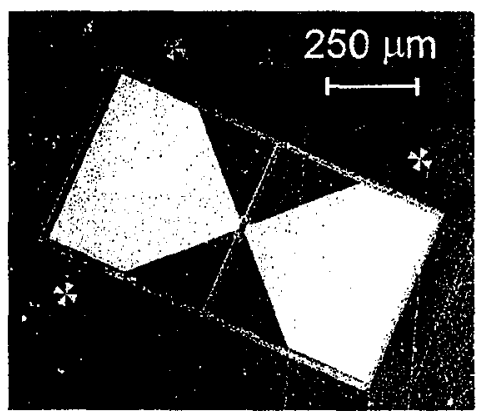

Fig. 1. Scanning electron micrographs of the fabricated samples: (a) wet etched CdMnTe wire (it was tilted slightly to obtain $3 \mathrm{D}$ perspective), trenches etched to the substrate are visible as darker areas; (b) 10 wet etched CdTe wires connected in parallel; (c) dry etched (Ar ion milling) PbTe wire, the sample was also tilted; (d) overall view of $\mathrm{GaAs} / \mathrm{GaAlAs}$ device, brighter areas correspond to AuGe ohmic contacts and alignment marks, separating grooves are also visible. The two-terminal wire (not seen) is located in the central part, between the contacts.

For II-VI group materials we used $\mathrm{Cd}_{x} \mathrm{Mn}_{1-x}$ Te:In films with $x=0$ or $x=1 \pm 0.1 \%$, with typical thickness of $0.3 \mu \mathrm{m}$, and electron concentrations 
around $10^{18} \mathrm{~cm}^{-3}$. They were grown by molecular beam epitaxy (MBE) onto (001) oriented semi-insulating (SI) GaAs epiready substrates with $10 \AA \mathrm{ZnTe}$ and $3 \mu \mathrm{m}$ CdTe undoped buffer layers. Detailed studies showed homogeneous impurity distribution and good structural properties of the epilayers [8]. For IV-VI group we used $\mathrm{PbTe}: \mathrm{Bi}$ films with thickness of $0.5 \mu \mathrm{m}$, grown onto $\mathrm{BaF}_{2}$ substrate (with $\mathrm{Pb}_{0.94} \mathrm{Eu}_{0.06} \mathrm{Te}$ buffer layer) and $\mathrm{PbSe}$ films with thickness of $1.7 \mu \mathrm{m}$, deposited directly onto $\mathrm{BaF}_{2}[9,10]$. Electron concentration ranged from $1 \times 10^{16}$ to $6 \times 10^{17} \mathrm{~cm}^{-3}$. Both II-VI and IV-VI devices were made using the single-level exposure (Fig. 1a, b, c).

The two-level electronolithography was used to fabricate short $(L=1.6 \mu \mathrm{m})$ two-terminal quantum wires from GaAs/GaAlAs heterostructures. First, the contact areas and alignment marks were exposed in $1 \times 1 \mathrm{~mm}$ writing field (Fig. 1d). After lift-off deposition and annealing of AuGe ohmic contacts the wires (in $100 \times 100 \mu \mathrm{m}$ area) and separating grooves were exposed. We used low mobility $\left(\approx 10^{5} \mathrm{~cm}^{2} /(\mathrm{V} \mathrm{s})\right)$ delta-doped heterostructure to make wires with $W_{\mathrm{geo}}=0.4,0.5$ and $0.6 \mu \mathrm{m}$. High mobility wire $\left(\approx 10^{6} \mathrm{~cm}^{2} /(\mathrm{V} \mathrm{s})\right)$ with $W_{\text {geo }}=0.4 \mu \mathrm{m}$ was made by Ismail et al. $[11,12]$. In both cases the wires were defined using wet mesa etching down to the GaAs substrate.

For high mobility sample the elastic mean free path $(l \approx 8 \mu \mathrm{m})$ was larger than wire length, whereas for low mobility GaAs/GaAlAs samples $(l \approx 0.6 \mu \mathrm{m})$ and $\mathrm{PbTe}$ samples $(l \approx 2 \mu \mathrm{m})$ the mean free path was comparable to distances between the contacts. For CdTe and CdMnTe wires $l$ was smaller $(\approx 0.01 \mu \mathrm{m})$. Therefore, from the point of view of elastic scattering electron transport in II-VI materials was diffusive.

\section{Universal conductance fluctuations in CdMnTe}

At non-zero temperatures not only elastic but also inelastic scattering occurs and destroys the phase coherence of electron waves. The inelastic scattering rate depends strongly on temperature as $\tau_{\phi} \propto T^{-p}(p=1 \div 2)$, and therefore only at low enough $T$ transport might be coherent over the entire sample. Following Landauer-Büttiker approach $[13,14]$ conductance $G$ of quasi-one-dimensional system is then given by

$$
G=\frac{e^{2}}{h} \sum_{i}^{N} t_{i}
$$

where $t_{i}=\sum_{j} t_{i j}$ and $t_{i j}$ is the transmission probability between channel $i$ and $j$ ( $N$ is the total number of channels). For large $N$ we have $t_{i} \approx l / L$, as predicted by random matrix theory [15] and confirmed experimentally [16]. If transport is coherent, transmission probabilities are influenced by the interference effects. Semiclassically we may write [17]:

$$
G \sim t_{i j} \sim \sum_{\alpha, \beta} \exp \left[\frac{\mathrm{i}}{\hbar}\left(S_{\alpha}-S_{\beta}\right)\right],
$$

where $S_{\alpha}, S_{\beta}$ are the classical action integrals over the interfering paths $\alpha$ and $\beta$. For given impurity configuration these cross-terms have arbitrary but fixed value since the energy is constant. Therefore any perturbation which affects the phase 
of the electron wave will cause these interference terms to vary in a complex way. Changing the carrier density or magnetic field will cause $G$ to fluctuate in a random (but reproducible) manner. Those variations in $G$ are called universal conductance fluctuations (UCF).

If transmission probabilities for different channels were independent, the amplitude of UCF would be given as $\Delta G \sim G / N \sim\left(e^{2} / h\right)(l / L)$ which, however, is in contradiction with the experimental results and diagrammatic calculations [18]. The important point is that $t_{i}$ 's are correlated and the effective number of independent channels is $N_{\text {eff }} \approx N(l / L)$ [19]. Therefore

$$
\Delta G \sim \frac{G}{N_{\text {eff }}} \sim \frac{e^{2}}{h}
$$

and fluctuations are "universal".

At non-zero temperature, $\Delta G$ is reduced when the "coherence volume" becomes smaller than the sample size. Scale over which phase is still maintained is called phase coherence length $L_{\phi}=\sqrt{D \tau_{\phi}}$, where $D$ is the diffusion constant. Similarly thermal diffusion length $L_{\mathrm{T}}=\sqrt{D \hbar / k_{\mathrm{B}} T}$ defines size over which coherence persists if a veraged thermally near Fermi energy.

Figure 2a presents conductance at $30 \mathrm{mK}$ as a function of the magnetic field for a CdTe:In wire with the electron concentration $n=1.0 \times 10^{18} \mathrm{~cm}^{-3}$. Aperiodic conductance fluctuations are well resolved and reproducible. It is interesting to compare these data to the magnetoconductance of 10 parallel wires of the same length $(L=5 \mu \mathrm{m})$ made of the same material (see insert). As expected, UCF are averaged over impurity configurations in each of the wires and no more resolved - only the weak localization conductance minimum around zero magnetic field is visible (the detailed discussion of magnetoconductance at weak magnetic fields is given by Jaroszyński et al. [20] in this volume). At $T=30 \mathrm{mK}$ we have $L_{\mathrm{T}}=$ $0.55 \mu \mathrm{m}$ and $L_{\phi}=12 \mu \mathrm{m}$ [5], therefore amplitude $\Delta G$ of the single wire should be reduced approximately by the factor $L_{\mathrm{T}} / L=0.11$, in good agreement with the experiment.

In Fig. 2b, the further reduction of $\Delta G$ with increasing temperature is shown - it corresponds to the shrinkage of "coherence volume" at higher $T$. Note, however, that the mean period of the fluctuations - the so-called correlation field $H_{\mathrm{c}}$ - is temperature independent.

A striking influence of magnetic impurities on the UCF is shown in Fig. 3, where data for $\mathrm{Cd}_{1-x} \mathrm{Mn}_{x}$ Te:In with $x=1 \pm 0.1 \%$ and $n=8 \times 10^{17} \mathrm{~cm}^{-3}$ are shown. $\Delta G$ is independent of the magnetic field and varies with the temperature similarly to $\mathrm{CdTe}$. This points to a minor efficiency of spin-disorder scattering, a conclusion consistent with a small density of states in semiconductors. In contrast to $\mathrm{CdTe}$, the fluctuation pattern exhibits a distinct field (Fig. 3a) and temperature (Fig. 3b) dependence. This new effect may have its origin in the spin splitting-induced redistribution of carriers between the spin subbands. The redistribution, and a corresponding shift of the Fermi energy $\varepsilon_{F}$ with respect to spin-up and spin-down subband edges, result in a gradual change of phase factors which contribute to the interference terms (2). Since in semimagnetic materials the spin splitting is proportional to the magnetization $M$ [21], the mean period of the fluc- 

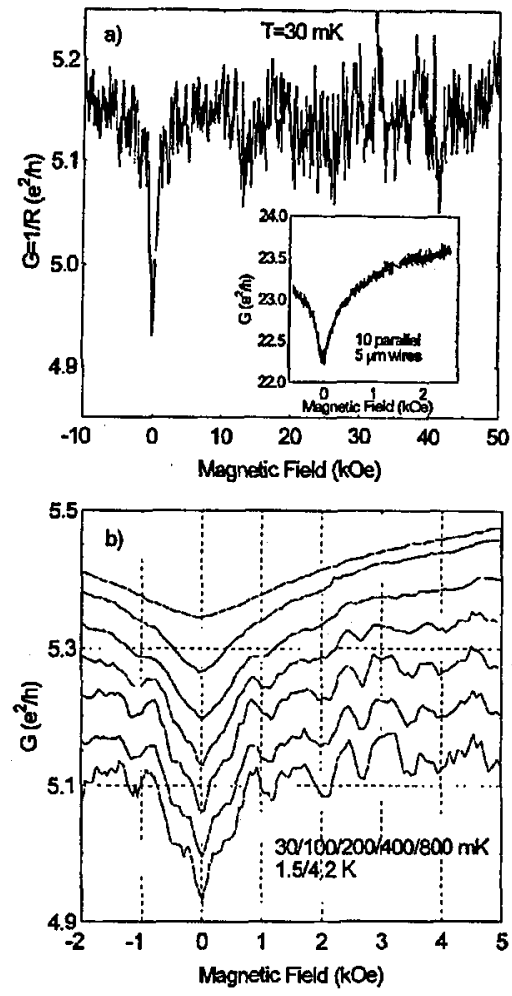

Fig. 2. (a) Conductance $G$ in universal units as a function of the magnetic field for the wire of $n^{+}$-CdTe at $30 \mathrm{mK}$. (b) Low field conductance fluctuations at various temperatures between $30 \mathrm{mK}$ and $4.2 \mathrm{~K}$ (traces for the lowest temperatures are shifted downwards). Inset shows conductance changes for 10 wire device (see Fig. 1b) at $30 \mathrm{mK}$.

tuations should be temperature independent if $G$ is plotted as a function of $M$. This is indeed the case - see inset to Fig. 3a. Furthermore, calculated value of the correlation field related to the spin splitting, agrees well with experiment [5].

\section{Strained PbTe and PbSe quantum wires}

The physical widths of CdTe and CdMnTe wires corresponded quite closely to its geometrical, lithographically defined cross-sections. This was not the case for $\mathrm{GaAs}$ and $\mathrm{PbTe} / \mathrm{PbSe}$ devices. For GaAs heterostructure wires $W_{\text {geo }}>W$ because of the depletion region occurring at the etched walls of the mesa structure. For IV-VI epilayers grown on $\mathrm{BaF}_{2}$ strain-induced effects come into play, modifying physical width of the electron system.

Figure 4 shows local and nonlocal resistance of $\mathrm{PbTe}$ wire with $W_{\text {geo }}=1 \mu \mathrm{m}$ as a function of temperature. Note the extremely large nonlocal component for $T \lesssim 200 \mathrm{~K}$. It was suggested that a difference between the thermal expansion coefficients of the substrate and the film below $200 \mathrm{~K}$ (which causes an overall biaxial tensile strain in the epilayer of the order of $1-2 \times 10^{-3}$ ) was responsible 

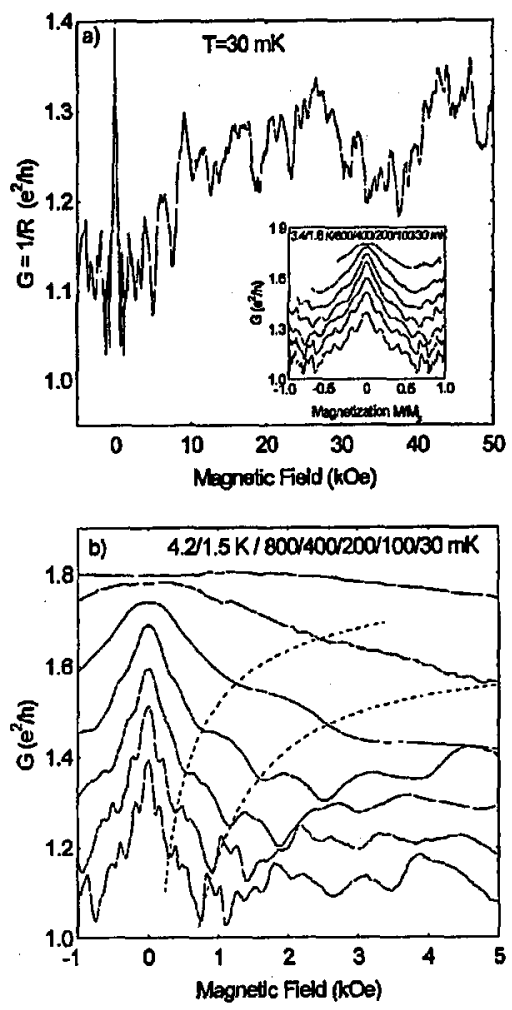

Fig. 3. Conductance changes as a function of the magnetic field for the wire of $n^{+}-\mathrm{Cd}_{1-x} \mathrm{Mn}_{x} \mathrm{Te}$ with $x=1 \%$ at $30 \mathrm{mK}(\mathrm{a})$ and at various temperatures between $30 \mathrm{mK}$ and $4.2 \mathrm{~K}$ (traces for the lowest temperatures are shifted downwards). Dotted lines are guides for the eye, and visualize a strong temperature dependence of the conductance features (b). Inset shows magnetoresistance data plotted as a function of magnetization (magnetization units are given in [5]).

for the observed transport anomalies $[10,6]$. This difference leads to the biaxial strain, whose magnitude varies substantially near the wire edges (see inset). The effect of such nonuniform strain on electron transport is particularly large in the studied system due to its large piezoresistivity associated with the strain-induced electron redistribution between the valleys. This model explains the existence of "edge channels" with the enhanced conductance and, in particular, the appearance of the nonlocal conductance below $200 \mathrm{~K}$.

The presence of edge channels were documented further by studies as a function of the line width and by a strong sensitivity of the conductance to the side-gate voltage as well as by large magnitude of both the UCF and the transverse magnetoresistance $[6,10]$. The strain effects present in IV-VI epilayers give a fascinating opportunity to construct high mobility quantum devices and study coherent transport. 


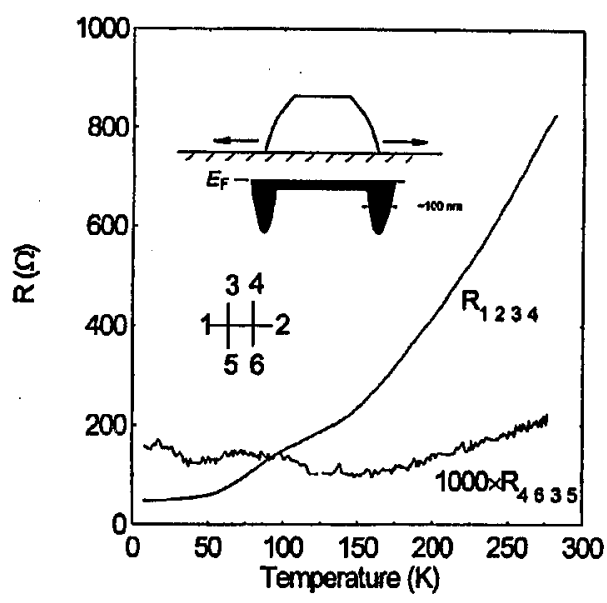

Fig. 4. Local and non-local resistance as a function of the temperature for the wire of PbTe (see Fig. 1c). Inset shows contact labels and explains schematically the origin of strain-induced edge conductivity. The cross-section of the deformed wire is shown under assumption that at low temperatures the substrate is less contracted than the etched structare. Resulting nonuniform strain may increase the electron concentration at the edges of a sample (below).

\section{Edge current strips in GaAs/GaAlAs wires}

For high mobility, ballistic wire we have $t_{i j}=\delta_{i j}$ and, including spin degeneracy, Eq. (1) becomes

$$
G=\frac{2 e^{2}}{h} N .
$$

This gives "quantized ballistic conductance" widely observed in GaAs heterostructure point contacts $[3,4]$. Typically a $2 \mathrm{D}$ electron gas in such systems is divided into two regions by a split gate constriction. As the contact width is varied a step-like dependence of $G$ with reasonably flat plateaus is observed.

It is commonly accepted that Eq. (4) is still valid in high magnetic fields, and magnetotransport of high mobility systems is usually described in terms of the edge current concept [22]. Semiclassically, in high enough field, $N$ is equal to the number of skipping cyclotron orbits flowing in one direction close to the wire edge. In this approach, edge currents have zero width, and its total number is always even. This model, however, neglects completely many electron effects. For example, the electron distribution across the wire is step-like (see Fig. 5) and the electron gas is incompressible - changing occupation of the subband leads to the change of the channel width. Screening of the confinement potential is not taken into account because incompressible electrons have a uniform density.

Recently, the one-electron picture of electron transport in quantum wires has been questioned. Chklovskii et al. [7] proposed a quantitative electrostatic theory of edge channels showing that the minimization of the many-electron energy of 


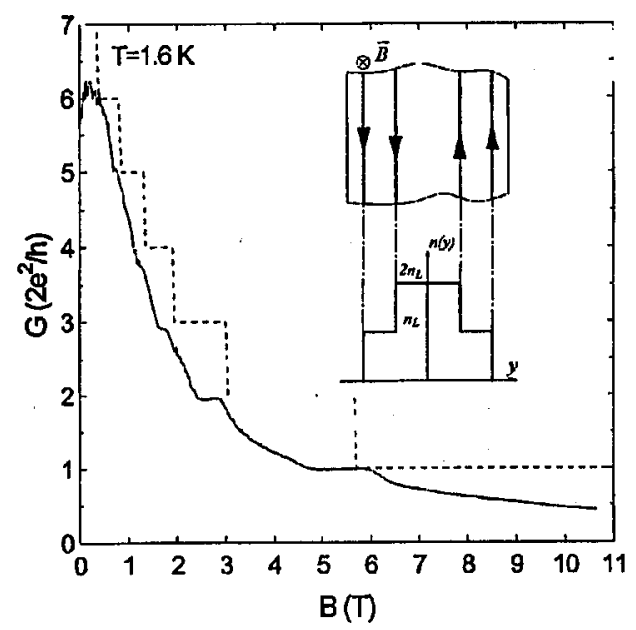

Fig. 5. Conductance $G$ vs. magnetic field $B$ for high mobility two-terminal wire of GaAs heterostructure with $W \approx 0.25 \mu \mathrm{m}$. $G$ is in $2 e^{2} / h$ units since the spin features are not resolved. Dashed line was calculated in one-electron approximation. Inset shows the top view of quantum wire and illustrates the concept of edge currents. The electron distribution across the wire ( $y$ direction) in one-electron model, when $G=2$, is shown below. $n_{\mathrm{L}}=e B / h$ is two-dimensional electron concentration of completely filled Landau level.

quantum wire in the high magnetic fields leads to the formation of finite width edge current "strips" in which the electron liquid is compressible. Additionally, they assumed that

$$
G=\frac{2 e^{2}}{h} \nu_{\mathrm{H}}
$$

where $\nu_{\mathrm{H}}$ is a filling factor at the center of the wire. More recently, Stoof and Bauer [23] extended this model by taking into account the exchange interaction among the electrons. We checked the validity of Eq. (5) and provided experimental evidences for edge current strips formation in narrow wires of GaAs heterostructure.

In Fig. 5 we compare our experimental data on the field dependence of the conductance to the theoretical predictions of one-electron theory. The confining potential of the wire has been assumed to be parabolic with the parameters determined from the weak-field depopulation of quasi-1D electron subbands $[12,24]$. Magnetic field quantizes the conductance and reasonably exact steps in $G$ of height $2 e^{2} / h$ are observed. The widths of the quantized plateaus are, however, much smaller than the ones predicted by Eq. (4). Figure 6 shows the data again together with the conductance obtained from Eq. (5) where $\nu_{\mathrm{H}}$ was calculated according to [7]. The parameters of the wire were taken from low-field one-electron approximation. A good agreement between theoretical predictions and experimental findings, attained with no adjustable parameters, indicates that indeed alternating strips of compressible and incompressible liquids are formed in strong magnetic 
fields (see inset). When the central strip in the channel is incompressible, the conductance $G$ is quantized in units of $2 e^{2} / h$. Ilowever, the plateaus of $G$ are very narrow, in good quantitative agreement with theory. The widths of plateaus are

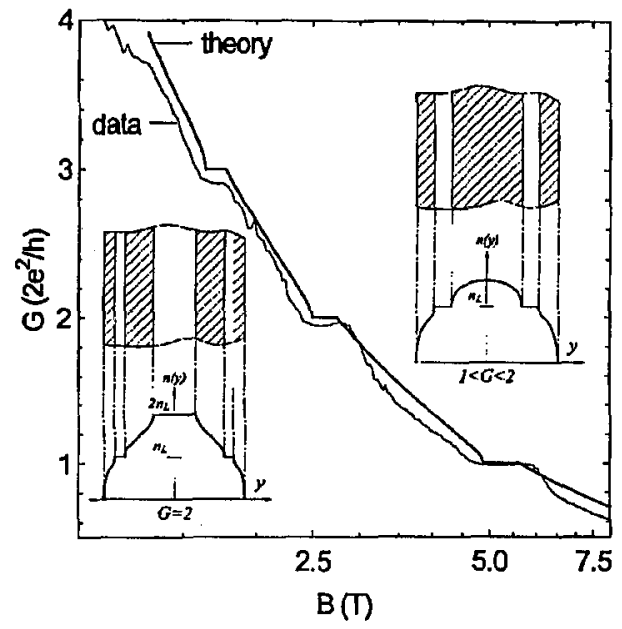

Fig. 6. Magnetoconductance data of Fig. 5 shown together with calculations performed according to the many-electron theory [7]. Inset illustrates the formation of incompressible and compressible (hatched) strips of electron liquid. When $G$ is quantized the central strip is incompressible and dielectric-like (left). For higher fields it disappears and compressible, metal-like central strip forms. In that case the electron density changes continuously across the wire (right).

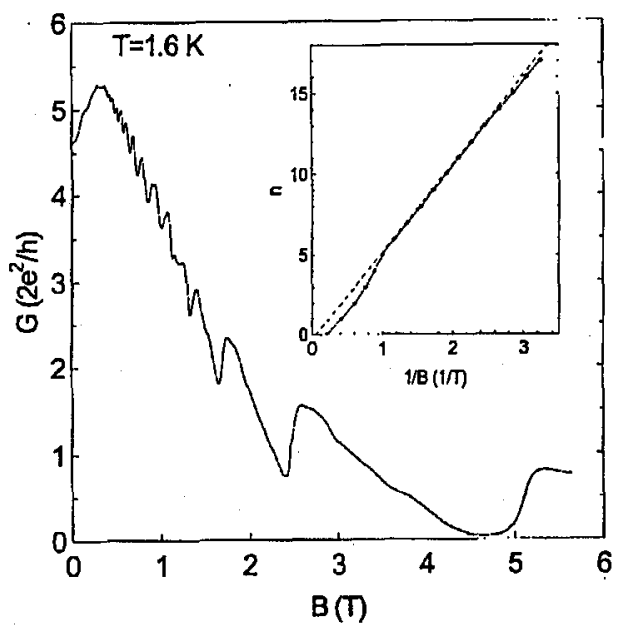

Fig. 7. Conductance vs. magnetic field for low mobility wire of GaAs heterostructure with $W \approx 0.45 \mu \mathrm{m}$. Inset shows the number of conductance minima vs. $1 / B$. Note the high field region where the phase of the oscillations changes. 
small because in a much wider range of the magnetic fields current strips in the center of the channel are compressible. In this range $G$ is not quantized.

Weak disorder may localize the low electron density edges of the compressible strip leading to the increase in plateau width, which was observed previously [12] and is also visible in Fig. 6. However, the presence of strong disorder may contribute to the localization of the entire compressible electron liquid.

Figure 7 shows magnetoconductance data for low mobility sample with geometrical width $W=0.6 \mu \mathrm{m}$ and physical width $W=0.45 \mu \mathrm{m}$. High field conductance minima are asymmetric and their positions are shifted relatively to the lower field Shubnikov-de Haas oscillations. For disordered sample the localization of the whole central current strip occurs in high enough magnetic field.

\section{Acknowledgments}

I would like to thank T. Dietl, G. Grabecki, J. Jaroszyński, F. Kuchar, E. Kamińska, E. Papis, A. Piotrowska, M. Sawicki and T. Skośkiewicz for essential contributions and helpful suggestions. This work was supported by the Committee for Scientific Research under contract No. PBZ-Z011/P4/93/01.

\section{References}

[1] See G. Owen, Rep. Prog. Phys. 48, 795 (1985); L. Veneklasen, in: Handbook of VLSI Microlithography, Eds. W.B. Glendinning, J.N. Helbert, Noyes Publications, Park Ridge 1991, p. 365; I. Brodie, J.J. Muray, The Physics of Micro/Nano-Fabrication, Plenum, New York 1992.

[2] See Mesoscopic Phenomena in Solids, Eds. B.L. Altshuler, P.A. Lee, R.A. Webb, Elsevier, Amsterdam 1991; S. Washburn, R.A. Webb, Rep. Prog. Phys. 55, 1311 (1992).

[3] B.J. van Wees, H. van Houten, C.W.J. Beenakker, J.G. Williamson, L.P. Kouwenhoven, D. van der Marel, C.T. Foxton, Phys. Rev. Lett. 60, 848 (1988).

[4] D.A. Wharam, M. Pepper, H. Ahmed, J.E.F. Frost, D.G. Hasko, D.C. Peacock, D.A. Ritchie, G.A.C. Jones, J. Phys. C 21, L209 (1988).

[5] J. Jaroszyński, J. Wróbel, M. Sawicki, E. Kamińska, T. Skośkiewicz, G. Karczewski, T. Wojtowicz, A. Piotrowska, J. Kossut, T. Dietl, Phys. Rev. Lett. 75, 3170 (1995); Surf. Sci., in press.

[6] G. Grabecki, J. Wróbel, T. Dietl, M. Sawicki, T. Skośkiewicz, E. Papis, E. Kamińska, A. Piotrowska, N. Frank, Y. Ueta, G. Sprinholz, G. Bauer, this volume; Superlatt. Microstruct., in press.

[7] D.B. Chklovskii, K.A. Matveev, B.I. Shklovskii, Phys. Rev. B 47, 12605 (1993).

[8] See G. Karczewski, A. Zakrzewski, M. Kutrowski, J. Jaroszyński, W. Dobrowolski, E. Grodzicka, E. Janik, T. Wojtowicz, J. Kossut, Acta Phys. Pol. A 87, 241 (1995).

[9] N. Frank, A. Voiticek, H. Clemens, A. Holtzinger, G. Bauer, J. Cryst. Growth 126, 293 (1993).

[10] G. Grabecki, S. Takeyama, S. Adachi, Y. Takagi, T. Dietl, E. Kaminiska, A. Piotrowska, E. Papis, N. Frank, G. Bauer, Jpn. J. Appl. Phys. 34, 4433 (1995).

[11] K.Y. Lee, D.P. Kern, D.P. Ismail, S. Washburn, J. Vac. Sci. Technol. B 9, 2834 (1991). 
[12] J. Wróbel, F. Kuchar, K. Ismail, K.Y. Lee, H. Nickel, W. Schlapp, G. Grabecki, T. Dietl, Surf. Sci. 305, 615 (1994); J. Wróbel, T. Brandes, F. Kuchar, B. Kramer, K. Ismail, K.Y. Lee, H. Hillmer, W. Schlapp, T. Dietl, Europhys. Lett. 29, 481 (1995).

[13] R. Landauer, Philos. Mag. 21, 863 (1970); see also R. Landauer, in: Analogies in Optics and Micro Electronics, Eds. W. van Haeringen, D. Lenstra, Kluwer, Dordrecht 1990, p. 243, and references therein.

[14] M. Büttiker, Phys. Rev. Lett. 57, 1761 (1986).

[15] I. Kander, Y. Imry, U. Sivan, Phys. Rev. B 41, 12941 (1990).

[16] J. Wróbel, F. Kuchar, K. Ismail, K.Y. Lee, W. Schlapp, Surf. Sci. 263, 261 (1992).

[17] M.C. Gutzwiller, Chaos in Classical and Quantum Mechanics, Springer, Berlin 1990, pp. 184, 283.

[18] P.A. Lee, A. Douglas Stone, H. Fukuyama, Phys. Rev. B 35, 1039 (1987).

[19] Y. Imry, Europhys. Lett. 1, 249 (1986).

[20] J. Jaroszyński, J. Wróbel, M. Sawicki, T. Skośkiewicz, G. Karczewski, T. Wojtowicz, J. Kossut, T. Dietl, E. Kamińska, E. Papis, A. Piotrowska, R. Duś, R. Nowakowski, Acta Phys. Pol. A 90, (1996), Proc. of this Conf. (Part II).

[21] See e.g. T. Dietl, in: IIandbook on Semiconductors, Ed. T.S. Moss, Vol. 3b, North-Holland, Amsterdam 1994, p. 1251.

[22] P. Streda, J. Kucera, A.H. McDonald, Phys. Rev. Lett. 59, 1973 (1987).

[23] T.I. Stoof, G.E.W. Bauer, Phys. Rev. B 52, 12143 (1995).

[24] J.F. Weisz, K.-F. Berggren, Phys. Rev. B 40, 1325 (1989). 